\title{
Prolonged Hyperperfusion in a Child With ATP1A2 Defect-Related Hemiplegic Migraine
}

\author{
Katherine Cobb-Pitstick (1D, Dana D. Cummings, Giulio Zuccoli
}

Keywords: Perfusion, ATP1A2, Hemiplegic migraine

doi:10.1017/cjn.2020.83

Can J Neurol Sci. 2020; 47: 687-688

A 5-year-old developmentally normal male presented with acute left-sided weakness, right-eye deviation, and agitation after right-sided head trauma. Exam was notable for agitation, inability to speak, right gaze preference, and left hemiparesis. Magnetic resonance imaging (MRI) head five hours after symptom onset demonstrated right hemispheric decreased perfusion, decreased caliber of the middle cerebral artery (MCA), posterior cerebral artery (PCA), and anterior cerebral artery (ACA), and hypooxygenated cortical veins (Figure 1A-D). MRI on day 2 demonstrated persistent decreased perfusion and improved but decreased caliber of the right distal MCA/ACA/PCA, and hypertonic saline infusion was begun. MRI on day 4 demonstrated hyperperfusion with increased caliber of the ACA and PCA. MRI on day 8 demonstrated continued right cerebral hyperperfusion and edema. MRI on day 13 demonstrated hyperperfusion and cortical edema with increased caliber of the right distal MCA/ACA/PCA arteries (Figure 1E-F).

Mental status and motor symptoms started improving on day 5. Neurologic exam at discharge (day 14) demonstrated persistent left-arm weakness and cognitive slowing. Genetic testing revealed a heterozygous variant (A297T: $889 \mathrm{G}>\mathrm{A}$ ) in ATPase $\mathrm{Na}+/ \mathrm{K}+$ transporting subunit alpha 2 (ATP1A2). In silico analyses predicting deleterious effects for this non-conservative amino acid substitution supported the conclusion that this was a novel pathologic gene variant.
Approximately 7 weeks following his presentation, he had persistent but improved left hemiparesis with decreased ability to resist movement, and pronator drift. Neuropsychology evaluation performed 10 weeks from presentation was notable for difficulty in many neurocognitive domains, including verbal reasoning and receptive language. At 10 months after presentation, parents felt that his left-arm functionality was back to baseline. At that time, he demonstrated persistent subtle left hemiparesis demonstrated by decreased left-arm swing with gait testing, but full strength to resistance testing. His academic performance had improved, and he had returned to school to repeat kindergarten.

This patient had post-traumatic hemiplegic migraine accompanying a novel gene defect in ATP1A2. He presented with hemispheric hypoperfusion followed by long-lasting hyperperfusion. Previous cases of hemiplegic migraine accompanying ATP1A2 gene defects demonstrated persistent cerebral edema 9 days $^{1}$ and 14 days after symptom onset. ${ }^{2}$ In our patient, the transition from hypoperfusion to hyperperfusion occurred 30 to 72 hours after symptom onset. Perfusion changes and cortical edema were still present on day 13. This is the longest MRI proven duration of perfusion changes in hemiplegic migraine known to these authors. The perturbation of cerebrovascular homeostasis for such a long duration and the prolonged clinical recovery time highlight a category of neurogenetic susceptibility to brain injury quite distinct from transient ischemic attack and stroke.

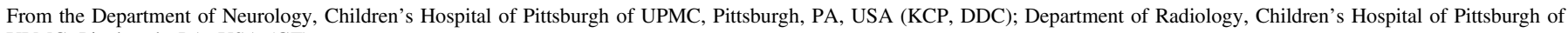
UPMC, Pittsburgh, PA, USA (GZ)

Received November 20, 2019. Final Revisions Submitted February 27, 2020. Date of Acceptance April 19, 2020.

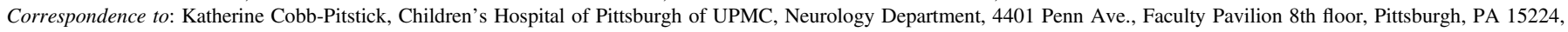
USA. Email: katherine.cobbpitstick@chp.edu 


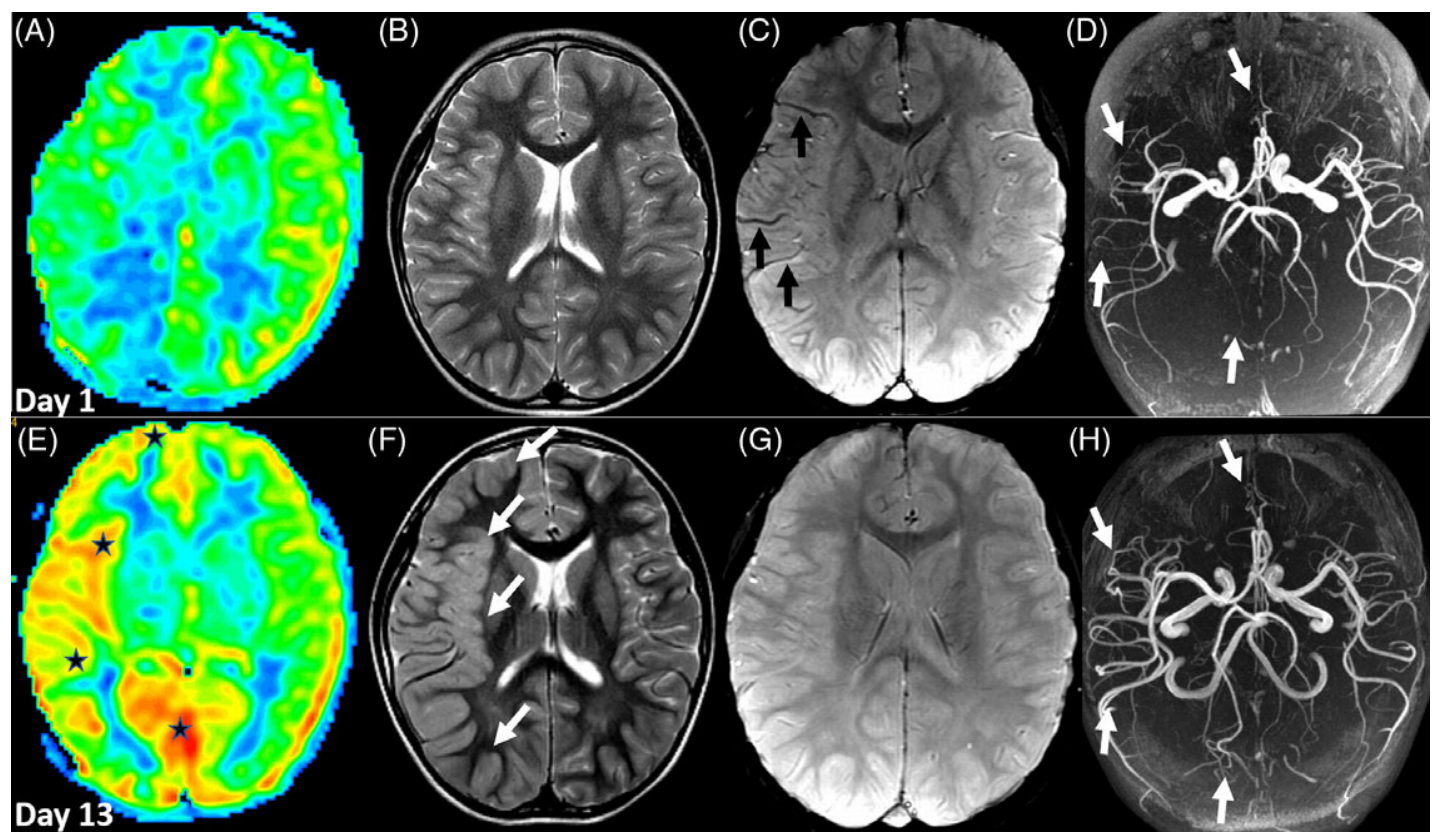

Figure 1: Day 1: Decreased perfusion in right cerebral hemisphere (A) without T2 changes (B); susceptibility weighted imaging (SWI) shows hypo-oxygenated blood within cortical veins of right cerebral hemisphere $(C)$; right MCA and PCA branches decreased in caliber $(D)$. Day 13: rebound hyperperfusion in right cerebral hemisphere (E); cortical edema involving right hemisphere $(F)$; normal $S W I(G)$; increased vessel caliber of anterior and posterior circulation $(H)$.

\section{DISCLOSURES}

Dr. Cummings received consult fees as a stroke consultant to the Vaccine Injury Compensation Program, US Department of Justice in 2017-2018. The other authors have no financial disclosures.

\section{STATEMENT OF AUTHORSHIP}

$\mathrm{KCP}$, DDC, and GZ were all involved with the drafting and editing of this manuscript.

\section{REFERENCES}

1. Dreier JP, Jurkatt-Rott K, Petzold GC, et al. Opening the blood-brain barrier preceding cortical edema in a severe attack of FHM type II. Neurology. 2005;64:2145-47.

2. Vanmolkot RKJ, Stroink H, Koenderink JB, et al. Severe episodic neurological deficits and permanent mental retardation in a child with a novel FHM2 ATP1A2 mutation. Ann Neurol. 2005;59: 310-14. 